\title{
Mechanisms of the apoptosis induced by CD176 antibody in human leukemic cells
}

\author{
BIN YI $^{1,2^{*}}$, MIN ZHANG ${ }^{1,2 *}$, REINHARD SCHWARTZ-ALBIEZ ${ }^{3}$ and YI CAO $^{1}$ \\ ${ }^{1}$ Laboratory of Molecular and Experimental Pathology, Key Laboratory of Animal Models and Human Disease Mechanism \\ of CAS and Yunnan Province, Kunming Institute of Zoology, Chinese Academy of Sciences, 32 Jiaochang Donglu, Kunming, \\ Yunnan 650223; ${ }^{2}$ Graduate University of the Chinese Academy of Sciences, 19A Yuquanlu, Beijing 100049, P.R. China; \\ ${ }^{3}$ Translational Immunology Unit, German Cancer Research Center, Im Neuenheimer Feld 280, Heidelberg 69120, Germany
}

Received December 17, 2010; Accepted February 11, 2011

DOI: $10.3892 /$ ijo.2011.992

\begin{abstract}
CD176 (Thomsen-Friedenreich antigen) is a tumorassociated carbohydrate structure. In a previous study, we observed that the anti-CD176 antibody can induce the apoptosis of CD176-positive leukemic cells. In this study, we investigated the mechanisms of apoptosis induced by the CD176 antibody. We found that CD95 (FAS, APO-1) and the death receptor 4 (DR4) (TRAIL-R1) are co-expressed with CD176 on the surface of defined leukemic cells as observed by confocal microscopy and flow cytometry analyses. Furthermore, CD95, CD45, CD43 and DR4 are carrier proteins of CD176 in hematopoietic cells recognized by means of sandwich solid-phase enzyme linked immunosorbent assay and co-immunoprecipitation. As shown by microarray analysis, 20 genes which are directly related to the execution, induction or positive regulation of apoptosis, were up-regulated after CD176 antibody treatment of the KG1 cell line. Nine differentially expressed genes observed in the microarray analysis were verified by quantitative real-time polymerase chain
\end{abstract}

Correspondence to: Professor Yi Cao, Key Laboratory of Animal Models and Human Disease Mechanism of Chinese Academy of Sciences and Yunnan Province, Kunming Institute of Zoology, Chinese Academy of Sciences, 32 Jiaochang Donglu, Kunming, Yunnan 650223, P.R. China

E-mail: caoy@mail.kiz.ac.cn

*Contributed equally

Abbreviations: mAb, monoclonal antibody; PAA, polyacrylamide; PBS, phosphate-buffered saline; TF, Thomsen-Friedenreich antigen; VCN, Vibrio cholerae neuraminidase (sialidase); q-RT-PCR, quantitative real-time polymerase chain reaction; ELISA, solid-phase enzyme linked immunosorbent assay; PMSF, phenylmethanesulfonyl fluoride; BSA, bovine serum albumin; SDS-PAGE, sodium dodecyl sulfate-polyacrylamide gel electrophoresis; OPD, o-phenylenediamine dihydrochloride; PI, propidium Iodide; PVDF, polyvinylidene difluoride

Key words: CD176, Thomsen-Friedenreich antigen, leukemia, apoptosis, signaling pathway reaction in the KG1 and MT4 cell lines. Six genes (DAXX, CASP3, CHUK, RIPK2, NFKBIA and DFFA) out of these nine are involved in five apoptotic pathways: The CD95 signaling pathway, the DR3 and DR4/5 death receptor pathway, caspase cascade in apoptosis, the mitochondrial signaling pathway, and apoptotic DNA fragmentation and tissue homeostasis. Thus, we hypothesized that the CD176 antibody binds to the CD176 carbohydrate structure present on apoptosisassociated glycoproteins, such as CD95 and DR4 at the cellular surface, which activates apoptotic pathways, and consequently results in the apoptosis of CD176-positive cells. CD176 is expressed at the surface of human leukemic cells, but is almost absent in normal and benign adult human tissues. Thus, CD176 could be a promising target for anti-tumor therapy based on the induction of tumor-specific apoptosis.

\section{Introduction}

The Thomsen-Friedenreich (TF) antigen is defined as the carbohydrate epitope (glycotope) sequence Gal $\beta 1-3 \mathrm{GalNc} \alpha 1-\mathrm{R}$ $(1,2)$. The TF antigen was assigned as CD176 during the Seventh Workshop and Conference on Human Leucocyte Differentiation Antigens (3). In this study, we use the CD designation for this oligosaccharide structure. CD176 is masked in normal and benign tissues by terminal sialylation (4), but it becomes exposed during tumorigenesis as a tumorspecific antigen (5). Approximately $70-80 \%$ of carcinomas carry CD176 on their surface (6). CD176 is also expressed in leukemia and lymphoma cells (7-9). There is evidence to suggest that CD176 could turn out to be a favorable prognostic marker in T-cell acute lymphoblastic leukemia $(10,11)$. In a previous study, we observed that the anti-CD176 antibody can induce the apoptosis of CD176-positive leukemic cells (12).

Apoptosis is an important regulatory process involved in the homeostasis and normal development of organisms. The suppression of apoptosis can result in many pathological disorders, such as cancer (13). Thus, promoting apoptosis may have the potential for effectively treating various cancers (14). One approach is to eradicate cancer cells by targeting tumorassociated carbohydrate antigens through the induction of antibodies which trigger apoptosis (15). As CD176 is strongly expressed on the surface of tumor cells and is virtually absent 
from normal adult human tissues (4), there is reason to assume that the carbohydrate structure provides a suitable determinant for cancer biotherapy through the induction of apoptosis. As a ubiquitous core structure, CD176 was found in a cryptic manner in many glycoproteins (12). We hypothesized that the apoptosis induced by the anti-CD176 antibody could be related to the apoptosis-associated glycoproteins on the cellular surface. It is known that CD95 (Fas, Apo-1), CD43, CD45, death receptor 4 (DR4) (TRAIL-R1) and DR3 (TRAMP) are apoptosis-associated glycoproteins on the surface of leukemic cells (16-18). In this study, we investigated i) whether CD176 is carried by distinct apoptosis-associated glycoproteins (CD95, CD45, CD43, DR4 and DR3), and ii) which signaling pathway is involved in the apoptosis induced by the CD176 antibody.

\section{Materials and methods}

Antibodies. The monoclonal antibodies (mAbs) employed in this study included CD95 mAb (DX2, mouse IgG1, Abcam, Hong Kong, China), DR4 mAb (DR-4-02, mouse IgG1, Abcam), DR3 mAb (JD3, mouse IgG1, BioLegend, San Diego, CA, USA), CD176 mAb (A78-G/A7, mouse IgM) (19), CD43 (DF-T1, Dako, Copenhagen, Denmark) and CD45 (HI30, BD Biosciences Pharmingen, Heidelberg, Germany). The CD95 polyclonal antibody (ab2437, rabbit IgG, Abcam) was also used.

Anti-CD176 serum was generated as follows: Mouse red blood cells (RBC), stored in Alserver's solution at $4^{\circ} \mathrm{C}$, were washed three times in phosphate-buffered saline (PBS). Erythrocytes were incubated with neuraminidase from Vibrio cholerae neuraminidase (VCN, N6514, Sigma, St. Louis, MO, USA) at a final concentration of $0.1 \mathrm{U} / \mathrm{ml}$ in PBS containing $\mathrm{Ca}^{2+}$ for $1 \mathrm{~h}$ at $37^{\circ} \mathrm{C}$ with occasional shaking. Thereafter, the cells were washed four times in PBS and prepared as $50 \%$ (v/v) cell suspension in PBS. The erythrocytes treated with VCN in this manner agglutinated strongly with amaranthus caudatus lectin (B-1255; Vector Laboratories, Inc., Burlingame, CA, USA) which preferentially binds to the terminal galactosyl $(\beta-1,3) \mathrm{N}$-acetylgalactosamine structure (TF antigen). BALB/c mice, 8-10 weeks of age, were injected intraperitoneally with $0.5 \mathrm{ml}$ or subcutaneously with $0.2 \mathrm{ml} \mathrm{RBC}$ pre-treated with VCN. Following three boost immunizations every 2 weeks, 50-100 $\mu \mathrm{l}$ of blood were collected by tail bleeding. The indirect solid-phase enzyme linked immunosorbent assay (ELISA) was used to estimate the titer of anti-CD176 antibody in the sera. When the antibody titer reached 1:1000, the sera were collected. In order to remove other interfering antibodies, the anti-CD176 sera were absorbed with equal volumes of untreated erythrocytes for at least $90 \mathrm{~min}$ at $4^{\circ} \mathrm{C}$ with occasional shaking. The mouse experiments were carried out according to the local and national law on the protection of animals and were approved by the local authorities.

Cell lines and cell culture. Human leukemia cell lines derived from acute myelogenous leukemia (KG1, KG1a, HL-60), erythroblastic cell leukemia (K562), pro-monocytic leukemia (U937), hairy cell leukemia (JOK-1), B-cell leukemia (Daudi), T-cell leukemia (CEM-C7, C8166, MT4) and acute monocytic leukemia (THP-1) were used in this study. All cell lines were cultured in RPMI-1640 medium (GIBCO Invitrogen, Grand
Island, NY, USA) with $10 \%$ fetal bovine serum and $100 \mathrm{U} / \mathrm{ml}$ of penicillin and streptomycin at $37^{\circ} \mathrm{C}$ in a $5 \% \mathrm{CO}_{2}$ incubator.

Flow cytometry analysis. The cell lines were stained at densities of $1 \times 10^{6}$ cells $/ 100 \mu \mathrm{l}$. They were washed twice with PBS containing $2 \%$ bovine serum albumin (BSA) and incubated with the appropriate primary $\mathrm{mAbs}$ at $4^{\circ} \mathrm{C}$ for $20 \mathrm{~min}$, followed by R-phycoerythrin ${ }^{+}$-conjugated goat anti-mouse IgG ( $\gamma$ chain-specific; 77753, Jackson ImmunoResearch Laboratories Inc., West Grove, PA, USA) or anti-IgM-FITC ( $\mu$ chain-specific; F9259, Sigma) at $4^{\circ} \mathrm{C}$ for $20 \mathrm{~min}$. After incubation, the cells were washed twice again and resuspended in PBS and 2\% glucose. Cells were analyzed on a FACScan flow cytometer (BD Biosciences, Rockville, MD, USA), collecting data of 10,000 cells for each histogram.

For the detection of masked CD176, the cells were incubated with VCN (N6514, Sigma) at a concentration of $20 \mathrm{mU} / \mathrm{ml}$ in medium for $60 \mathrm{~min}$ at $37^{\circ} \mathrm{C}$ to remove NeuAc in the $\alpha 2-3$, $\alpha 2-6$, and $\alpha 2-8$ linkages. Then, flow cytometry analysis was performed as described above.

Immunofluorescence staining. The cells were grown on sterile polylysine (Sigma)-coated slides in culture medium overnight, the medium was carefully aspirated and the slides were air-dried. For immunofluorescence staining, the cells were fixed in acetone at $-20^{\circ} \mathrm{C}$ for $10 \mathrm{~min}$, followed by blocking with $2 \% \mathrm{BSA}$ for $30 \mathrm{~min}$, and incubated with the mAbs for $1 \mathrm{~h}$ at room temperature (RT). After washing with PBS, the slides were incubated with a mixture of fluorescein isothiocyanate (FITC)-conjugated anti-mouse $\operatorname{IgM}$ ( $\mu$ chain-specific; F9259, Sigma) and CyTM3-conjugated goat anti-mouse IgG ( $\gamma$ chain-specific; 69732, Jackson ImmunoResearch Laboratories Inc.) for $1 \mathrm{~h}$ in a dark chamber. Following three washing steps, the cells were stained with DAPI (Beyotime Biotechnology Co. Ltd., Shanghai, China) for $5 \mathrm{~min}$. Then the slides were mounted with glycerol and analyzed by confocal laser scanning microscopy (Zeiss LSM510 Meta, Germany) and fluorescence microscopy (Leica DM 2500, Germany).

Co-immunoprecipitation. Target cells were lysed in ice-cold RIPA lysis buffer [50 mM Tris- $\mathrm{HCl} \mathrm{pH} 8.0,150 \mathrm{mM} \mathrm{NaCl}$, $1 \%$ Nonidet P-40, $0.5 \%$ sodium deoxycholate, $0.1 \%$ sodium dodecyl sulfate (SDS)] containing phenylmethanesulfonyl fluoride (PMSF) at a final concentration of $5 \mathrm{mmol} / \mathrm{l}$ and a cocktail of proteinase inhibitors (set III, Merck KGaA, Darmstadt, Germany) with dilution at 1:100, and homogenized with gentle agitation at $4^{\circ} \mathrm{C}$ for $30 \mathrm{~min}$. The extracts were centrifuged at $12,000 \mathrm{rpm}$ for $20 \mathrm{~min}$. The supernatant was taken and incubated with the capture CD95 mAb, CD45 mAb, or CD43 mAb at $4^{\circ} \mathrm{C}$ overnight with mild shaking. Then $15 \mu \mathrm{l}$ of protein G-Sepharose beads (P4691, Sigma) were added, and the incubation was continued for $5 \mathrm{~h}$ at $4^{\circ} \mathrm{C}$ with gentle rotation. Immune complexes were washed four times in lysis buffer and suspended in 2X SDS-polyacrylamide gel electrophoresis (PAGE)-sample buffer, and then boiled for $10 \mathrm{~min}$. The samples were separated by SDS-PAGE and transferred to polyvinylidene difluoride (PVDF) membranes, and were then analyzed by Western blot analysis using anti-CD176 Ab (IgM) and peroxidase-labeled goat anti-mouse $\operatorname{IgM}$ antibody as described previously. 
ELISA. An indirect ELISA was performed to estimate the titer of anti-CD176 antibody in the sera from the immunized mice. Microplates were coated with $50 \mu \mathrm{l} /$ well of $\mathrm{TF} \alpha$ polyacrylamide (PAA) (Syntesome, Munich, Germany) at a concentration of $2 \mu \mathrm{g} / \mathrm{ml}$ in carbonate/bicarbonate buffer (pH 9.6), and incubated overnight at $4^{\circ} \mathrm{C}$. After washing three times with $0.05 \%$ Tween-20 in PBS, the remaining binding sites were blocked with $2 \%$ BSA in PBS (200 $\mu \mathrm{l} /$ well) for $2 \mathrm{~h}$ at RT. The anti-CD176 mouse sera (see above) were serially diluted with $1 \%$ BSA in PBS. After incubation with the antiCD176 sera for $2 \mathrm{~h}$ at RT and three further washing steps, the wells were treated with $50 \mu \mathrm{l}$ of peroxidase-labeled goat antimouse antibody (Southern Biotech, Birmingham, AL, USA) for $60 \mathrm{~min}$ at $\mathrm{RT}$. The color reaction was developed with o-phenylenediamine dihydrochloride (OPD) solution at RT. The reaction was stopped with $2.5 \mathrm{M}$ sulfuric acid. Negative controls were performed with $1 \%$ BSA instead of the serum. The absorbance was measured at $492 \mathrm{~nm}$.

For the analysis of carrier molecules of CD176, a sandwich ELISA was used: Ninety-six well polystyrene microplates were coated with the capture antibodies against CD95, CD45, CD43, or DR4 at a concentration of $1-5 \mu \mathrm{g} / \mathrm{ml}$ in carbonate/ bicarbonate buffer (3.30 g/l Na$\left.{ }_{2} \mathrm{CO}_{3} 6.0 \mathrm{~g} / 1 \mathrm{NaHCO}_{3} \mathrm{pH} 9.6\right)$ at $4^{\circ} \mathrm{C}$ for $14 \mathrm{~h}$. After blocking the remaining protein-binding sites in the coated wells by adding $200 \mu 15 \%$ non-fat dry milk or $5 \% \mathrm{BSA}$ overnight at $4^{\circ} \mathrm{C}, 100 \mu \mathrm{l}$ of the appropriately diluted supernatants of the cell lysates (described above) were added and incubated at RT for $2 \mathrm{~h}$. The plates were then incubated with CD176 mAb for $2 \mathrm{~h}$ at RT, followed by peroxidaselabeled goat anti-mouse IgM antibody ( $\mu$ chain-specific; Southern Biotech, Birmingham, AL, USA) for $1 \mathrm{~h}$ at RT. The color reaction was similar to the indirect ELISA (described above). Negative controls included 2\% BSA instead of the capture mAbs, cell lysates, detection mAb and secondary antibody (20).

Induction of apoptosis with CD176 antibody in vitro. The leukemia cells were plated onto a 12 -well plate at a concentration of $1 \times 10^{7}$ cells/well, and the cells were either treated with VCN at a concentration of $0.1 \mathrm{U} / \mathrm{ml}$ in culture medium for $60 \mathrm{~min}$ at $37^{\circ} \mathrm{C}$, or they were left untreated. After washing three times, the treated and untreated cells were incubated with the sterile anti-CD176 sera at serial dilutions (1:10 and 1:20) for $6,12,24$ and $48 \mathrm{~h}$ at $37^{\circ} \mathrm{C}$, respectively. The annexin V-FITC Apoptosis Detection Kit (KGA107; KeyGen, Nanjing, China) and propidium iodide (PI) were used to assess the apoptotic effects of the anti-CD176 serum. Negative controls were performed with normal mouse serum. The binding of annexin V or PI was quantitatively analyzed using a FACScan flow cytometer as described above.

Microarray hybridization and data analysis. The SBCPhalanx human one array ${ }^{\mathrm{TM}}$ oligo microarray containing 3096860 mer-oligo probes and 1081 quality control probes was used. Microarray hybridization was performed by the Shanghai Biochip Co., Ltd. (SBC). Briefly, total RNAs from KG1 cells treated with VCN and anti-CD176 serum (undergoing apoptosis $50 \%$ apoptotic cells) and the control KG1 cells without anti-CD176 serum treatment were extracted and purified by the Qiagen RNeasy mini kit (Qiagen, Hilden,
Germany). Total RNA (5 $\mu \mathrm{g}$ ) was amplified using the Amino Allyl MessageAmp ${ }^{\mathrm{TM}}$ Kit (Ambion, Austin, TX, USA). For hybridization, $10 \mu \mathrm{g}$ of RNA were labeled with Cyanine 5 fluorescent and mixed with hybridization buffer (Amersham Biosciences AB, Uppsala, Sweden), and then subjected to microarray hybridization. Hybridization was performed on slides for $16 \mathrm{~h}$ at $50^{\circ} \mathrm{C}$ in a dark humidity chamber, and the slides were washed with pre-warmed $2 \mathrm{X}$ SSPE buffer, $0.1 \%$ SDS solution for 2 mins at $42^{\circ} \mathrm{C}$. The slides were scanned at $10 \mu \mathrm{m}$ resolution with dual-wavelength scanner (Agilent Technologies, Santa Clara, CA, USA), and the images were processed with Imagen for fluorescence intensity acquisition. The microarray data were normalized using GeneSpring (Silicon Genetics, Redwood City, CA, USA) software. The data from the two replicates were averaged. The data were first filtered to include only apoptosis-associated genes that had a detection signal in four samples. Secondly, a gene was defined as differentially expressed if the intensity ratio (Cy5) was exhibited to increase or decrease over 2-fold in four experimental results, and finally, the intensity ratio (Cy5) had to show the same direction of changes (up- regulated or down-regulated) in all four experimental results. The SBC analysis system (http:// sas.ebioservice.com/) was used to identify the apoptotic pathways induced by the CD176 antiserum and to calculate the statistical significance of each pathway. The analysis system assigns these differential genes to the pathways by first matching the genes to similar genes in known pathways in the Biocarta database. Pathways with FDR-corrected P-values of $<0.001$ were considered to be statistically significant.

Quantitative real-time polymerase chain reaction ( $q-R T-P C R)$. q-RT-PCR based on SYBR-Green was performed to verify the results of the microarray analysis. Total RNA from KG1 and MT4 treated with VCN and the anti-CD176 serum as well as the control KG1 and MT4 with VCN and without the antiCD176 serum treatment was extracted as described above. The RNA was reverse-transcribed by M-MLV reverse transcriptase (Promega Corp., Madison, WI, USA). The expressions of the 12 genes that were related to the apoptosis from the microarray analysis were determined by q-RT-PCR (SYBR-Green I method). GAPDH was employed as the internal control, and distilled water was used as the negative control. The amplification reaction contained 10X PCR buffer, and 1.25 U JumpStart Taq (Sigma), $10 \mathrm{pmol}$ forward and reverse primers, $0.2 \mu \mathrm{mol}$ dNTP, $100 \mathrm{ng}$ template, and 0.2X SYBR-Green I (Amresco Inc., Cleveland, USA), in a final volume of $50 \mu$ l. The q-RT-PCR parameters were as follows: $30 \mathrm{sec}$ at $95^{\circ} \mathrm{C}$, and then 40 cycles of $10 \mathrm{sec}$ at $95^{\circ} \mathrm{C}, 30 \mathrm{sec}$ at $60^{\circ} \mathrm{C}$. Each sample was tested in triplicate. The reactions were carried out by the StepOne ${ }^{\mathrm{TM}}$ Real-Time PCR System (Applied Biosystems, CA, USA). The expressions of the 12 genes mRNA were normalized against GAPDH using the $2^{-\triangle \Delta C T}$ method (21). The primer sequences of GAPDH and the 12 genes were retrieved from PrimerBank (http://pga.mgh.harvard.edu/primerbank/).

\section{Results}

Expressions of CD176, CD95, CD45, CD43, DR4 and DR3 in leukemia cells. Flow cytometry analysis and immunofluorescence staining revealed that CD176, CD95, CD45, 
Table I. Comparison of reactivities of the anti-CD95, anti-CD45, anti-CD43, anti-DR4 and anti-DR3 monoclonal antibodies on various hematopoietic cell lines after sialidase treatment by flow cytometric analysis.

\begin{tabular}{|c|c|c|c|c|c|c|c|c|c|c|c|c|c|c|c|c|}
\hline \multirow[b]{2}{*}{ Cells } & \multicolumn{2}{|c|}{ CD43 } & \multicolumn{2}{|c|}{ CD45 } & \multicolumn{2}{|c|}{ CD95 } & \multicolumn{2}{|c|}{ CD95-CD176 } & \multicolumn{2}{|c|}{ DR3 } & \multicolumn{2}{|c|}{ DR3-CD176 } & \multicolumn{2}{|c|}{ DR4 } & \multicolumn{2}{|c|}{ DR4-CD176 } \\
\hline & NO & $\mathrm{VCN}$ & NO & $\mathrm{VCN}$ & NO & $\mathrm{VCN}$ & NO & $\mathrm{VCN}$ & NO & $\mathrm{VCN}$ & NO & $\mathrm{VCN}$ & NO & $\mathrm{VCN}$ & NO & $\mathrm{VCN}$ \\
\hline K562 & 80 & $0 \downarrow \downarrow \downarrow$ & 89 & $94 \uparrow$ & 1 & $8 \uparrow$ & 0 & 0 & 0 & 0 & 0 & 0 & 75 & $88 \uparrow$ & 44 & $88 \uparrow \uparrow$ \\
\hline KG1 & nd & nd & nd & nd & 40 & $90 \uparrow \uparrow$ & 22 & $90 \uparrow \uparrow \uparrow$ & 0 & 0 & 0 & 0 & 0 & 0 & 0 & 0 \\
\hline KGla & 98 & $37 \downarrow \downarrow \downarrow$ & 67 & $98 \uparrow \uparrow$ & 3 & $56 \uparrow \uparrow$ & nd & nd & nd & nd & nd & nd & nd & nd & nd & nd \\
\hline HL60 & nd & nd & nd & nd & 19 & $82 \uparrow \uparrow \uparrow$ & 0 & $67 \uparrow \uparrow \uparrow$ & 0 & 0 & 0 & 0 & 0 & 0 & 0 & 0 \\
\hline C8166 & nd & nd & nd & nd & 98 & $97 \downarrow$ & 75 & $97 \uparrow$ & 0 & 0 & 0 & 0 & 4 & $5 \uparrow$ & 6 & $2 \downarrow$ \\
\hline CEM-C7 & 80 & $12 \downarrow \downarrow \downarrow$ & 96 & $97 \uparrow$ & 67 & $83 \uparrow$ & nd & nd & nd & nd & nd & nd & nd & nd & nd & nd \\
\hline MT4 & nd & nd & nd & nd & 96 & $72 \downarrow$ & 13 & $72 \uparrow \uparrow$ & 0 & 0 & 0 & 0 & 19 & $36 \uparrow$ & 4 & $29 \uparrow \uparrow$ \\
\hline U937 & 98 & $98 \downarrow$ & 98 & $99 \uparrow$ & 98 & $98 \uparrow$ & nd & nd & nd & nd & nd & nd & nd & nd & nd & nd \\
\hline THP1 & nd & nd & nd & nd & 2 & $19 \uparrow$ & 0 & $11 \uparrow$ & 14 & $23 \uparrow$ & 2 & $34 \uparrow \uparrow$ & 0 & 0 & 0 & 0 \\
\hline JOK1 & 90 & $8 \downarrow \downarrow \downarrow$ & 88 & $95 \uparrow$ & 87 & $94 \uparrow$ & nd & nd & nd & nd & nd & nd & nd & nd & nd & nd \\
\hline Daudi & nd & nd & nd & nd & 25 & $70 \uparrow \uparrow$ & 10 & $38 \uparrow$ & 8 & $17 \uparrow$ & 10 & $2 \downarrow$ & 95 & $64 \downarrow$ & 95 & $64 \downarrow \downarrow$ \\
\hline
\end{tabular}

NO, untreated; VCN, treated with neuraminidase from Vibrio cholerae; nd, not done. $\downarrow$, Mean fluorescence intensity reduction of $<30 \%$; $\downarrow$, mean fluorescence intensity reduction of 30-60\%; $\downarrow \downarrow$, mean fluorescence intensity reduction of $>60 \%$. Mean fluorescence intensity reduction (mean fluorescence intensity for mAbs - mean fluorescence intensity for mAbs after VCN)/mean fluorescence intensity for mAbs. $\uparrow$, Mean fluorescence intensity increase of $<30 \%$; $\uparrow$, mean fluorescence intensity increase of 30-60\%; $\uparrow \uparrow \uparrow$, mean fluorescence intensity increase of $>60 \%$. Mean fluorescence intensity increase (mean fluorescence intensity for mAbs after VCN - mean fluorescence intensity for mAbs)/mean fluorescence intensity for mAbs.

CD43, DR4 and DR3 were differently expressed in the leukemia cell lines (Table I). KG1, K562, C8166, MT4, THP1 and Daudi were positive for CD176. All leukemia cells examined expressed CD95, but the expression values of K562, THP-1 and KG1a were very low. K562, C8166, MT4 and Daudi expressed DR4 at varying intensities. Only THP1 and Daudi cells were stained by anti-DR $3 \mathrm{mAb}$.

The reactivities of the mAbs were compared with and without VCN pre-treatment in order to see whether epitopes recognized by the $\mathrm{mAb}$ are dependent on the presence of terminal sialic acid residues (Table I). The pre-treatment of VCN resulted in an increase of CD95 reactivity in most of the cell lines except for C8166 and MT4. The reaction of antiCD45 was increased, while the binding of anti-CD43 was decreased after the treatment with VCN. It could be that the conformation epitopes of CD43 glycoprotein including peptide backbone with oligosaccharides are disrupted by VCN treatment.

Immunofluorescence double staining was performed to investigate the co-expression of CD95 and DR4 with CD176 in leukemia cell lines. Flow cytometry experiments revealed semi-quantitative co-expression data as presented in Fig. 1 and Table I. A partial overlay of CD176 surface expression with that of DR4 and CD95 was observed by confocal microscopy (Fig. 2).

To evaluate whether the co-expression of DR4, DR3 and CD95 with CD176 was affected after VCN pre-treatment, we performed semi-quantitative flow cytometric experiments and found that the co-expression of DR3 and CD176 was increased in THP-1, but decreased in Daudi cells (Table I). The coexpression of DR4 and CD176 in K562 and MT4 was increased while the co-expression was decreased in C8166 and Daudi (Fig. 1). Remarkably, the cell lines THP-1 and HL60 which were negative for the co-expression of CD95 and CD176 became positive after $\mathrm{VCN}$ pre-treatment. The co-expression of CD95 and CD176 on KG1, MT4 and HL60 was increased by $>60 \%$ (Fig. 1). However, that of CD95 and CD176 in THP-1, C8166 and Daudi was only slightly increased (>10\%).

CD95, CD45, CD43, and DR4 carry CD176 in leukemia cells. In order to identify the carrier proteins for the CD176 carbohydrate sequence, we analyzed nine leukemic cell lines by immunoprecipitation and sandwich ELISA. The glycoproteins of CD45, CD43 and CD95 were immunoprecipitated from the lysates of the three cell lines and detected by Western blot analysis with CD176 mAb (Fig. 3).

In a sandwich ELISA with anti-CD45, anti-CD43, antiCD95 and anti-DR4 as the catcher antibodies and CD176 $\mathrm{mAb}$ as the detection antibody, captured CD95, CD45 and CD43 were found to react with the anti-CD176 in five cell lines (KG1a, U937, K562, JOK-1 and CEM-C7), but captured DR4 was only bound by anti-CD176 in K562 and Daudi (data not shown). These data indicate that CD95, CD45, CD43 and DR4 are carrier proteins of CD176 in leukemia cells (Table II).

Differential expression of apoptosis-related genes by microarray analysis. In order to assess the pathways involved in the apoptosis induced by the CD176 antibody, we used gene microarray analysis to analyze the differential expressions of genes after the CD176 antiserum treatment. The apoptotic effect of the CD176 antiserum treatment was investigated by 

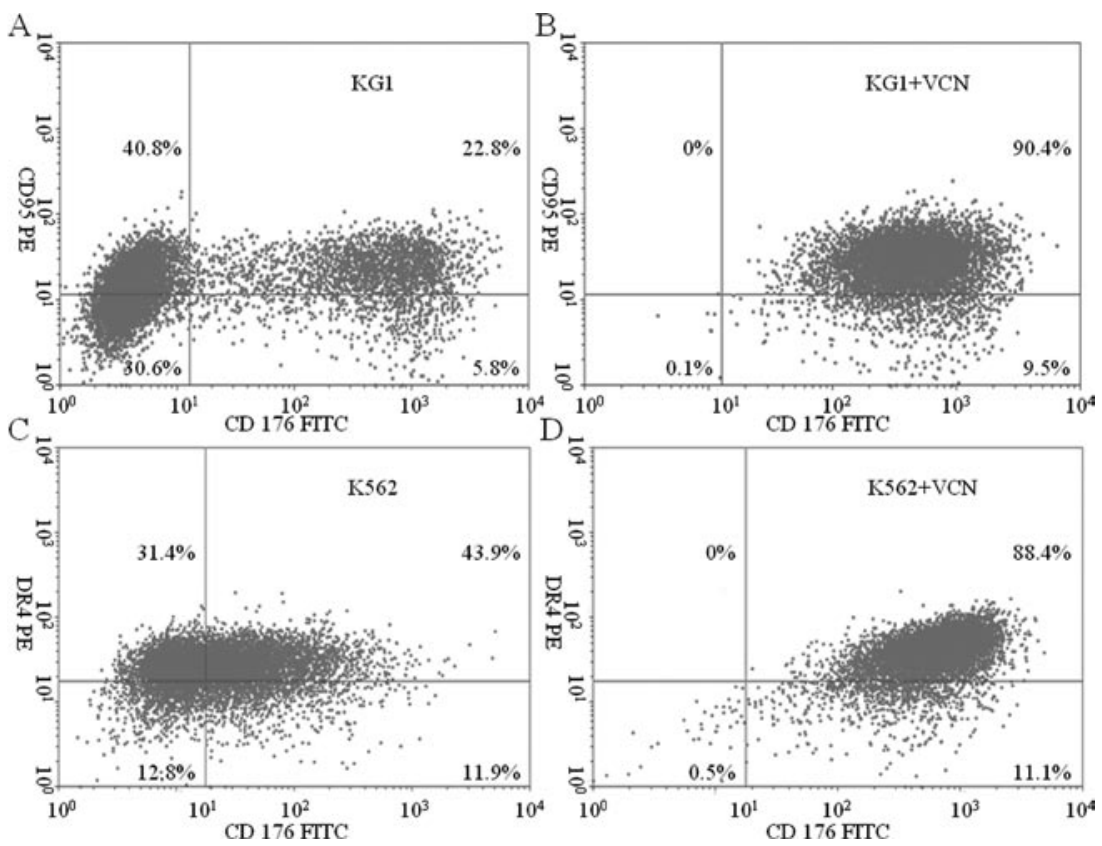

Figure 1. (A and B) Flow cytometric analysis of $\mathrm{CD} 95^{+} / \mathrm{CD} 176^{+}$expression in $\mathrm{KG} 1$ cells before and after neuraminidase treatment. (C and D) Flow cytometric analysis of $\mathrm{DR}^{+} / \mathrm{CD}_{176}{ }^{+}$expression in $\mathrm{K} 562$ cells before and after neuraminidase treatment. Neuraminidase treatment resulted in an increase in the percentage of KG1 cells expressing both CD95 and CD176 and of K562 cells expressing both DR4 and CD176.

A

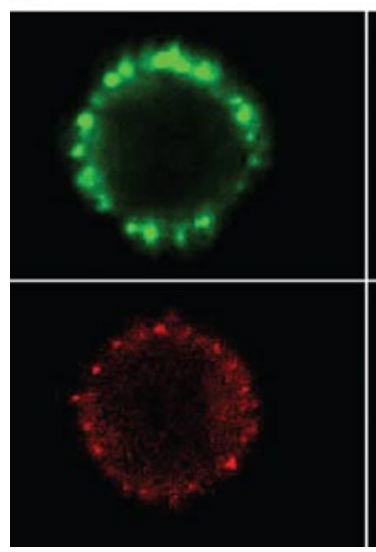

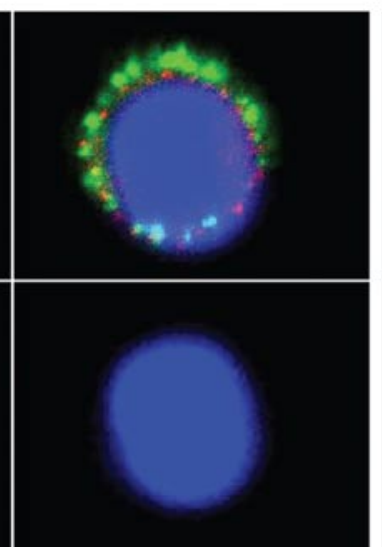

B

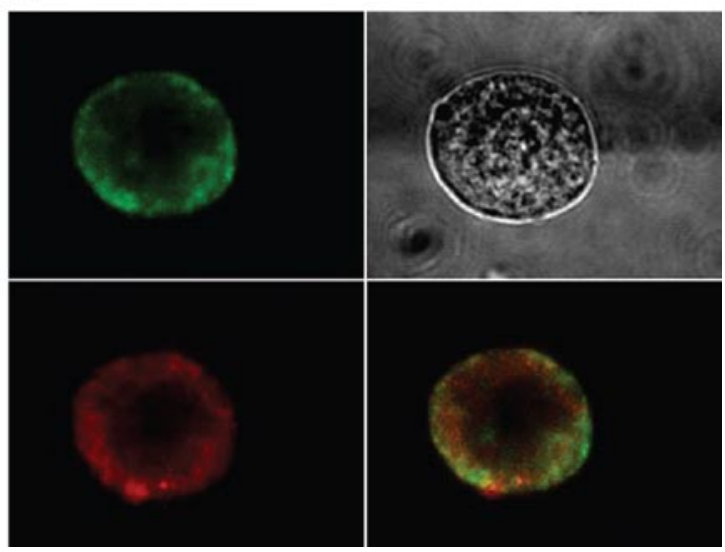

Figure 2. Double immunofluorescence staining of hematopoietic cells. (A) KG1 cell line was stained with CD95 mAb (DX2, mouse IgG1) and with CD176 mAb (A78-G/A7, mouse IgM), followed by incubation with anti-IgG-Cy3 ( $\gamma$ chain-specific, red) and anti-IgM-FITC ( $\mu$ chain-specific, green). (B) Daudi cell line was stained with DR4 mAb (DR-4-02, mouse IgG1) and with CD176 mAb (A78-G/A7, mouse IgM), followed by incubation with anti-IgG-Cy3 ( $\gamma$ chain-specific, red) and anti-IgM-FITC ( $\mu$ chain-specific, green). CD176 was co-expressed with CD95 or DR4. Magnification, x1000.

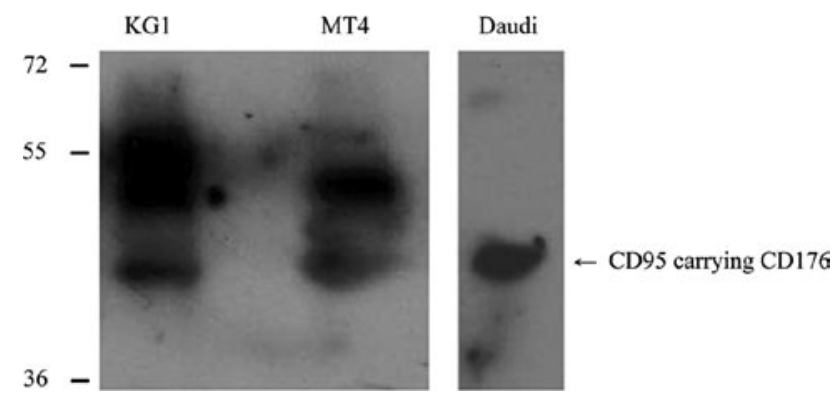

Figure 3. Immunoprecipitation of lysates from cell lines KG1, MT4 and Daudi. The CD95-immunoprecipitated samples were separated by SDS-PAGE and analyzed by immunoblotting using anti-CD176 Ab (IgM) and peroxidaselabeled goat anti-mouse $\operatorname{IgM}$ antibody. The results show that CD95 is a carrier of CD176 in human leukemic cells. annexin $\mathrm{V}$ staining and flow cytometry analysis. The percentage of KG1 cells bound to annexin V was about $30 \%$ after exposure to the CD176 antiserum, and this increased to about $60 \%$ after VCN pre-treatments followed by incubation with the CD176 antiserum. The genes with a $>2$-fold difference in expression after induction with the CD176 antiserum were defined as differentially expressed. Among those, 35 genes were involved in apoptosis (partial data shown in Tables III and IV). Of the 35 apoptosis-related genes, 27 were upregulated and 8 were down-regulated. Among the up-regulated genes, 15 genes were involved in the positive regulation of apoptosis, and 6 participated in the induction, execution and regulation of apoptosis. However, 6 up-regulated genes played roles in the negative regulation of apoptosis. Of the down- 
Table II. The results of the sandwich ELISA assays carried out to evaluate carrier molecules of CD176 using anti-CD95, anti-CD45 and anti-CD43 monoclonal antibodies as catchers in various hematopoietic cells.

\begin{tabular}{|c|c|c|c|c|c|c|c|c|c|c|c|c|c|c|c|c|c|}
\hline \multirow{2}{*}{$\begin{array}{l}\text { Catcher } \\
\text { antibody }\end{array}$} & \multicolumn{2}{|c|}{ KG1a } & \multicolumn{2}{|c|}{ KG1 } & \multicolumn{2}{|c|}{ U937 } & \multicolumn{2}{|c|}{ K562 } & JOK-1 & \multicolumn{2}{|c|}{ Daudi } & \multicolumn{2}{|c|}{ CEM-C7 } & \multicolumn{2}{|c|}{ MT4 } & \multicolumn{2}{|c|}{ C8166 } \\
\hline & NO & $\mathrm{VCN}$ & NO & VCN & NO & $\mathrm{VCN}$ & NO & VCN & NO VCN & NO & VCN & NO & VCN & NO & VCN & NO & VCN \\
\hline CD95 & + & ++ & + & + & - & ++ & + & ++ & ++ & + & + & + & ++ & + & + & + & + \\
\hline CD45 & + & + & nd & nd & + & + & + & + & + & nd & nd & + & + & nd & nd & nd & nd \\
\hline CD43 & - & + & nd & nd & - & + & + & ++ & + & nd & nd & + & ++ & nd & nd & nd & nd \\
\hline
\end{tabular}

NO, untreated; VCN, treated with neuraminidase from Vibrio cholerae; nd, not done. Scoring according to OD490 minus blank as follows: ++, $>0.5 ;+, 0.1-0.5 ;-,<0.1$.

Table III. Up-regulated apoptosis-related genes in KG1 after treatment with CD176 antibody and sialidase.

\begin{tabular}{llrl}
\hline GenBank ID & Symbol & Ratio & \multicolumn{1}{c}{ Biological process } \\
\hline NM_020529.1 & NFKBIA & 13.13 & Positive regulation of apoptosis \\
NM_018370.1 & FLJ11259 & 6.24 & Positive regulation of apoptosis \\
NM_003998.2 & NFKB1 & 3.72 & Anti-apoptosis \\
NM_003821.4 & RIPK2 & 3.33 & Induction of apoptosis \\
NM_003804.2 & RIPK1 & 3.04 & Positive regulation of apoptosis \\
NM_020532.4 & RTN4 & 2.93 & Positive regulation of apoptosis \\
NM_001350.3 & DAXX & 2.86 & Induction of apoptosis \\
NM_004323.2 & BAG1 & 2.84 & Anti-apoptosis \\
NM_001278.2 & CHUK & 2.79 & Positive regulation of apoptosis \\
NM_020418.2 & PCBP4 & 2.58 & Induction of apoptosis \\
NM_004346.2 & CASP3 & 2.39 & Execution of apoptosis \\
NM_133328.2 & DEDD2 & 2.17 & Induction of apoptosis \\
\hline
\end{tabular}

Table IV. Down-regulated apoptosis-related genes in KG1 after treatment with CD176 antibody and sialidase.

\begin{tabular}{llll}
\hline GenBank ID & Symbol & Ratio & \multicolumn{1}{c}{ Biological process } \\
\hline NM_003327.2 & TNFRSF4 & 0.15 & Negative regulation of apoptosis \\
NM_001223.2 & CASP1 & 0.17 & Positive regulation of apoptosis \\
NM_033046.1 & RTKN & 0.3 & Negative regulation of anti-apoptosis \\
NM_001197.3 & BIK & 0.32 & Induction of apoptosis \\
NM_004401.2 & DFFA & 0.36 & Negative regulation of apoptosis \\
NM_003820.2 & TNFRSF14 & 0.44 & Positive regulation of apoptosis \\
\hline
\end{tabular}

regulated genes, 4 genes were involved in the induction and positive regulation of apoptosis and in the negative regulation of anti-apoptosis. The other 4 down-regulated genes took part in the negative regulation of apoptosis. In summary, the differentially expressed genes are mainly related to the five apoptotic pathways in the apoptotic cells induced by the CD176 antibody: i) DAXX, CASP3, RIPK2 and DFFA in the CD95 signaling pathway, ii) CHUK, NFKBIA, NFKB1, CASP 3 and DFFA in the DR 3 and DR4/5 pathway, iii) CASP3, CASP1 and DFFA in caspase cascade pathway, iv) CASP3, DFFA, BAG1 and BIK in the mitochondrial pathway, and v) CASP3 and DFFA in the DNA fragmentation and tissue homeostasis pathway.

We selected the 12 differentially expressed genes by microarray analysis, and used q-RT-PCR to confirm their expressions in KG1 and MT4 induced by the CD176 antiserum. There were indeed consistent results concerning the nine genes in a comparison between microarray and q-RT-PCR data (Table VI).

Possible pathways involved in the apoptosis induced by the CD176 antibody. The relationship between the apoptotic 
Table V. Relationship between the apoptotic pathways and the differentially expressed genes found in the microarray analysis.

\begin{tabular}{|c|c|c|c|c|c|}
\hline Biocarta pathway ${ }^{a}$ & $\begin{array}{l}\text { No. of } \\
\text { related genes }\end{array}$ & $\begin{array}{l}\text { No. of } \\
\text { altered genes }\end{array}$ & Percentage & P-value & Q-value \\
\hline $\begin{array}{l}\text { Apoptotic DNA fragmentation } \\
\text { and tissue homeostasis }\end{array}$ & 9 & 2 & 22.22 & 0.0 & 0.0 \\
\hline Caspase cascade in apoptosis & 23 & 2 & 8.7 & 0.0002 & 0.0 \\
\hline FAS signaling pathway & 30 & 4 & 13.33 & 0.0 & 0.0 \\
\hline DR3 and DR4/5 death receptor pathway & 29 & 4 & 13.79 & 0.0 & 0.0 \\
\hline Mitochondrial signaling pathway & 21 & 3 & 14.29 & 0.0 & 0.0 \\
\hline
\end{tabular}

P-value $<0.001$ and Q-value (false discovery rate) $<0.001$ were considered to be significantly associated with the apoptotic pathway. The analysis of enrichment statistics is based on the SBC analysis system, http://sas.ebioservice.com/. all biocarta pathways were retrieved from: http://www.biocarta.com/genes/Apoptosis.asp.

Table VI. Comparison of the expressions of the 12 genes between the microarray and q-RT-PCR analyses.

\begin{tabular}{|c|c|c|c|}
\hline \multirow{2}{*}{$\begin{array}{l}\text { Apoptosis-related } \\
\text { genes }\end{array}$} & \multicolumn{2}{|c|}{ q-RT-PCR } & \multirow{2}{*}{$\frac{\text { Microarray }}{\text { KG1 }}$} \\
\hline & KG1 & MT4 & \\
\hline NFKBIA & $\uparrow \quad(1.9)$ & $\uparrow \uparrow \quad(2.7)$ & $\uparrow \uparrow \uparrow \uparrow(13.13)$ \\
\hline RIPK2 & $\uparrow \quad(1.7)$ & $\uparrow \uparrow \quad(2.3)$ & $\uparrow \uparrow \uparrow \quad(3.33)$ \\
\hline DAXX & $\uparrow \quad(1.7)$ & $\uparrow \uparrow \quad(2.3)$ & $\uparrow \uparrow \quad(2.86)$ \\
\hline CHUK & $\uparrow \uparrow \quad(2)$ & $\uparrow \uparrow \quad(2.6)$ & $\uparrow \uparrow \quad(2.79)$ \\
\hline CASP3 & $\uparrow \uparrow \uparrow(3.5)$ & $\uparrow \quad(1.5)$ & $\uparrow \uparrow \quad(2.39)$ \\
\hline PCBP4 & $\uparrow \uparrow \quad(2.7)$ & $\uparrow \quad(1.5)$ & $\uparrow \uparrow \quad(2.58)$ \\
\hline DEDD2 & $\uparrow \uparrow \quad(2)$ & $\uparrow \uparrow \uparrow \uparrow(4.7)$ & $\uparrow \uparrow \quad(2.17)$ \\
\hline BIK & $\uparrow \uparrow \uparrow \uparrow(4)$ & $\uparrow \uparrow \uparrow \uparrow(6.5)$ & $\downarrow \downarrow \downarrow \quad(0.32)$ \\
\hline DFFA & $\downarrow \downarrow \quad(0.5)$ & $\downarrow \downarrow \quad(0.7)$ & $\downarrow \downarrow \downarrow(0.36)$ \\
\hline CASP1 & $\downarrow \downarrow \quad(0.5)$ & $\downarrow \downarrow \quad(0.56)$ & $\downarrow \downarrow \downarrow \downarrow(0.17)$ \\
\hline NFKB1 & $\downarrow \downarrow \quad(0.52)$ & $\downarrow \downarrow \quad(0.7)$ & $\uparrow \uparrow \uparrow \quad(3.72)$ \\
\hline RIPK1 & $\downarrow \downarrow \downarrow \downarrow(0.22)$ & $\uparrow \quad(1.5)$ & $\uparrow \uparrow \uparrow(3.04)$ \\
\hline
\end{tabular}

The fold-change values of the 12 genes mRNA were normalized against GAPDH using the $2^{-\triangle \triangle C T}$ method. The changes in KG1 or MT4 cells treated with the CD176 antibody and the neuraminidase versus the control KG1 or MT4 cells treated only with the neuraminidase are indicated by arrows: $\downarrow, \downarrow \downarrow, \downarrow \downarrow \downarrow \downarrow$ stand for 25-50, 50-75 and 75-95\% decreased expressions, respectively. $\uparrow, \uparrow \uparrow, \uparrow \uparrow \uparrow, \uparrow \uparrow \uparrow \uparrow$ stand for $100-200,200-300,300-400$ and $>400 \%$ increased expressions, respectively.

pathways and the differentially expressed genes found on the microarray and q-RT-PCR tests was calculated by analysis of enrichment statistics. The results are summarized in Table V. Five possible apoptotic pathways were significantly involved in the apoptosis induced by the CD176 antibody (Fig. 4): i) The CD95 signaling pathway, ii) the DR3 and DR4/5 death receptor pathway, iii) caspase cascade in apoptosis, iv) the mitochondrial apoptotic signaling pathway, v) apoptotic DNA fragmentation and tissue homeostasis.

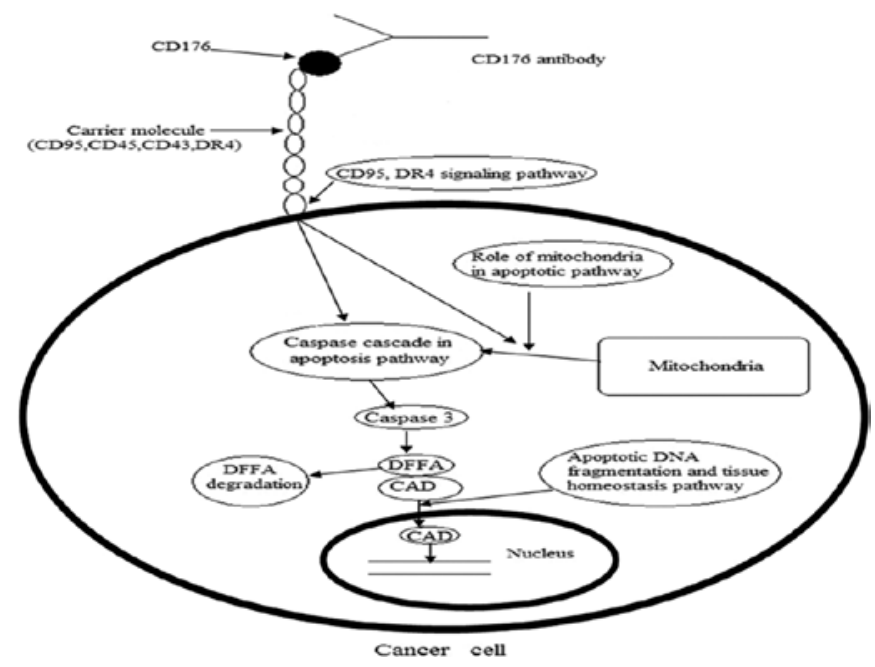

Figure 4. Schematic diagram summarizing the effect of apoptosis induced by the CD176 antibody.

\section{Discussion}

The induction of apoptosis is an approach of cancer biotherapy. For instance, trastuzumab which triggers the apoptosis of HER2-positive breast cancer cells has been used for clinical treatment (22). Natural carbohydrate-reactive IgM Abs capable of mediating the apoptosis of tumor cells are regarded as a mechanism of innate immune surveillance against cancer cells (23). In a previous study, we found that the CD176 antibody could trigger the apoptosis of $\mathrm{CD}_{176^{+}}$cancer cells (12) indicating that this carbohydrate structure offers a possibility for cancer biotherapy. However, the mechanisms underlying this apoptotic effect are not known. To this end, knowledge of the mechanisms of apoptosis mediated by the CD176 antibody is important for the development of a therapeutic approach.

CD176 (TF, Gal $\beta 1-3$ GalNAc $\alpha 1-R)$ is an ubiquitous core structure (core-1) found in a cryptic manner in many membrane glycoprotein, and sialic acid is one of the most important molecules for the masking of CD176. We assumed that the apoptosis mediated by the CD176 antibody may affect apoptotic 
signaling through surface expressed glycoproteins. Thus, the expression of the TF antigen (CD176) and apoptosis-associated glycoproteins (CD95, CD45, CD43, DR4 and DR3) was systematically investigated in several human leukemia cell lines using specific mAbs in our study. The following results were obtained: i) CD95 and DR4 were co-expressed with CD176 on the surface of defined leukemic cells as shown by confocal microscopy and flow cytometry analysis, ii) the binding of anti-CD95, anti-CD45, anti-DR4 and anti-DR3 mAbs can be enhanced after pre-treatment with $\mathrm{VCN}$, which demonstrates that these apoptosis-associated molecules, such as CD95 are sialoglycoproteins, iii) CD95, CD45, CD43 and DR4 are carrier proteins of CD176 in hematopoietic cells, as shown by sandwich ELISA and co-immunoprecipitation. From these data, the conclusion can be drawn that CD176 is carried by CD95, CD45, CD43 and DR4, and that the anti-CD176 antibody can bind these sialoglycoproteins.

The cell surface sialoglycoprotein, CD95, is a member of the tumor necrosis factor superfamily. The CD95 ligand or anti-CD95 monoclonal antibody can induce the CD95mediated apoptosis of human B-and T-cells and the removal of terminal sialic acids by sialidase enhances the apoptosis (16). The CD43 glycoprotein is abundantly expressed by hematopoietic cells (24). A number of studies have shown that the engagement of CD43 induces the apoptosis of T-cells and hematopoietic progenitor cells $(17,25)$. CD45, originally known as the leukocyte common antigen, is a type 1 transmembrane protein. The cross-linking of the extracellular portion of CD45 with monoclonal antibodies can induce apoptosis in $\mathrm{T}$-and B-cells and eosinophils (18). The level of O-glycosylation of B- or T-lymphocyte surface glycoprotein receptors, including CD43 and CD45 can regulate the apoptosis induced by galectin-1 $(26,27)$ or lectin jacalin $(28)$. DR4 is a pro-apoptotic receptor for the cytotoxic ligand, TRAIL, or the anti-DR4 monoclonal antibody (29). Apoptosis mediated by natural carbohydratereactive IgM Abs in humans has been reported $(23,30)$. In this study, we used the anti-CD176 serum gained by immunization of mice with RBC treated with $\mathrm{VCN}$ in order to confirm that the anti-CD176 antibody had indeed an apoptotic effect on CD176-positive leukemia cells, and further that the apoptosisassociated glycoproteins, such as CD95, DR4, CD45 and CD43 are carrier molecules of CD176 in hematopoietic cells. Based on previous results from other studies and ours, we hypothesized that the binding of the CD176 carbohydrate structure on apoptosis-associated glycoproteins by the anti-CD176 antibody can activate apoptotic glycoproteins and pathways, and then result in the apoptosis of CD176-positive cells.

The mechanisms underlying the apoptosis induced by the CD176 antibody on human leukemic cells was further investigated by microarray and q-RT-PCR analyses. Microarray analysis showed that 35 apoptosis-related genes were differentially expressed on KG1 with or without the treatment with the CD176 antibody. The 20 genes directly related to the execution, induction and positive regulation of apoptosis were up-regulated. These observations confirm that the anti-CD176 antibody can induce apoptosis. The microarray transcription data were verified for the 12 genes by q-RT-PCR assays on KG1 and MT4 cell lines. General trends of the expressions of the nine out of the 12 genes were consistent on the microarray and q-RT-PCR analyses. Six genes (DAXX, CASP3, CHUK,
RIPK2, NFKBIA and DFFA) out of the nine genes were significantly involved in five apoptotic pathways: i) The CD95 signaling pathway, ii) the DR3 and DR4/5 death receptor pathway, iii) caspase cascade in apoptosis, iv) the mitochondrial apoptotic signaling pathway, and v) apoptotic DNA fragmentation and tissue homeostasis (Fig. 4). The six genes together participated in the cross-talk of the five apoptotic pathways. DAXX, RIPK2, CASP3 and DFFA participated in the CD95 signaling pathway, and CHUK, NFKBIA, CASP3 and DFFA were involved in the apoptotic pathway of the DR3 and DR4/5 death receptors. The analysis of enrichment statistics demonstrated that the CD95 signaling pathway and the DR3 and DR4/5 death receptor pathway were mostly related to the apoptosis induced by the CD176 antibody. These results were expected. In the analysis of CD176 carrier molecules, we found that CD95 and DR4 glycoproteins are carrier molecules of CD176. The activation of caspase 3 and degradation of DFFA, which were early events during the apoptotic process, were involved in both the extrinsic (CD95, DR3 and DR4 death receptor) and intrinsic (mitochondrial) pathways. Crosslinking of O-glycosylated CD43 by anti-CD43 mAbs increased the CD95-induced apoptosis through the induction of CD95 aggregation in Jurkat T-cells (31). Galectin-1, a $\beta$-Gal binding protein, can induce T-cell apoptosis through the activation of caspase 3 (32). Thus, we hypothesized that the mechanisms of the apoptosis induced by the CD176 antibody and galectin-1 may be similar in hematopoietic cells. The CD176 antibody binds the carbohydrate structures in apoptosis-associated glycoproteins at the cellular surface (such as CD95, DR4, etc.), activates apoptotic pathways (such as FADD and caspase 8), triggers the activation of caspase 3, and induces DNA fragmentation as well as apoptosis.

The mechanisms by which the CD176 antibody activates apoptotic pathways are not yet clear. It is known that the CD95 ligand or an agonistic anti-CD95 antibody binding cell surface CD95 induces CD95 oligomerization and the formation of CD95 clusters resulting in caspase activation (33). Carbohydrate structures in apoptosis-associated glycoproteins also take part in the activation of the apoptotic pathways. The human IgM antibody, SAM-6, which binds to an O-linked carbohydrate moiety can result in tumor-specific apoptosis $(34,35)$. The O-glycosylation of DR4 and DR5 promotes the ligand-stimulated clustering of DR4 and DR5, which mediates the recruitment and activation of the apoptosis-initiating protease caspase 8 (36). In our study, the expression levels of CD95, DR4, CD45 and CD43 were not significantly changed after CD176 antibody treatment. Therefore, we hypothesized that the binding of the CD176 antibody to the carbohydrate structure in apoptosis-associated cell surface glycoproteins induces the clustering of the apoptosis-related proteins, and subsequently activates caspase pathways. Certainly, this has to be confirmed in future studies.

In conclusion, the mechanisms of apoptosis induced by the CD176 antibody could be that the CD176 antibody binds the CD176 carbohydrate structure in apoptosis-associated glycoproteins, such as CD95 and DR4, and then activates apoptotic pathways resulting in the apoptosis of CD176-positive cells. CD176 is expressed on the surface of human leukemic cells, but is almost absent in normal and benign adult human tissues (4). CD176 could be a promising target for apoptotic therapy. 


\section{Acknowledgements}

This study was financially supported by grants from the Yunnan Province Science and Technology Department (2008CCO15), the Chinese Academy of Sciences (KSCX2YW-R-196), and the National Natural Science Foundation of China (No. 81072563).

\section{References}

1. Clausen $\mathrm{H}$ and Hakomori S: ABH and related histo-blood group antigens; immunochemical differences in carrier isotypes and their distribution. Vox Sang 56: 1-20, 1989.

2. Kim Z and Uhlenbruck G: Untersuchungen über T-Antigen und T-Agglutinin. Z Immunol Forsch 130: 88-99, 1966.

3. Cao Y, Karsten U and Schwartz-Albiez R: Expression of ThomsenFriedenreich-related carbohydrate antigens on human leukemia cells. In: Leucocyte Typing VII. Mason D, et al (eds). Oxford University Press, Oxford, pp204-205, 2002.

4. Cao Y, Stosiek P, Springer GF and Karsten U: ThomsenFriedenreich-related carbohydrate antigens in normal adult human tissues: a systematic and comparative study. Histochem cell Biol 106: 197-207, 1996.

5. Springer GF: T and Tn, general carcinoma autoantigens. Science 224: 1198-1206, 1984.

6. Springer GF: Immunoreactive $\mathrm{T}$ and $\mathrm{Tn}$ epitopes in cancer diagnosis, prognsis and immunotherapy. J Mol Med 75: 594-602, 1997.

7. Muroi K, Suda T, Nakamura M, et al: Expression of sialosyl-Tn in colony-forming unit-erythroid, erythroblasts, $\mathrm{B}$ cells, and a subset of $\mathrm{CD}^{+}{ }^{+}$cells. Blood 83: 84-91, 1994.

8. Aller CT, Kucuk O, Springer GF and Gilman-Sachs A: Flow cytometric analysis of $\mathrm{T}$ and $\mathrm{Tn}$ epitopes on chronic lymphocytic leukemia cells. Am J Hematol 52: 29-38, 1996.

9. Inoue M, Nakada H, Tanaka $\mathrm{N}$ and Yamashina I: Tn antigen is expressed on leukosialin from T-lymphoid cells. Cancer Res 54 85-88, 1994

10. Kaspers GJ, Veerman AJ, Van Wering ER, et al: Prognostic significance of peanut agglutinin binding in childhood acute lymphoblastic leukemia. Leukemia 10: 675-681, 1996.

11. Veerman AJ, Hogeman PH, Huismans DR, Van Zantwijk CH and Bezemer PD: Peanut agglutinin, a marker for T-cell acute lymphoblastic leukemia with a good prognosis. Cancer Res 45: 1890-1893, 1985

12. Cao Y, Merling A, Karsten U, et al: Expression of CD175 (Tn) CD175s (sialosyl-Tn) and CD176 (Thomsen-Friedenreich antigen) on malignant human hematopoietic cells. Int J Cancer 123: 89-99, 2008.

13. Thompson CB: Apoptosis in the pathogenesis and treatment of disease. Science 267: 1456-1462, 1995.

14. Fesik SW: Promoting apoptosis as a strategy for cancer drug discovery. Nat Rev Cancer 5: 876-885, 2005.

15. Ravindranath NM, Nishimoto K, Chu K and Shuler C: Cellsurface expression of complement restriction factors and sialyl Lewis antigens in oral carcinoma: relevance to chemo-immunotherapy. Anticancer Res 20: 21-26, 2000.

16. Peter ME, Hellbardt S, Schwartz-Albibez R, et al: Cell surface sialylation plays a role in modulating sensitivity towards APO-1mediated apoptotic cell death. Cell Death Differ 2: 163-171, 1995.

17. Park WS, Chae JS, Jung KC, Choi WJ, Kook MC and Bae Y: Production and the characterization of monoclonal antibody against CD43, K06. Tissue Antigens 63: 46-53, 2004.
18. Blaylock MG, Sexton DW and Walsh GM: Ligation of CD45 and the isoforms CD45RA and CD45RB accelerates the rate of constitutive apoptosis in human eosinophils. J Allergy Clin Immunol 104: 1244-1250, 1999.

19. Karsten U, Butschak G, Cao Y, Goletz S and Hanisch FG: A new monoclonal antibody (A78-G/A7) to the Thomsen-Friedenreich pan-tumor antigen. Hybridoma 14: 37-44, 1995.

20. Lin WM, Karsten U, Goletz S, Cheng RC and Cao Y: Expression of CD176 (Thomsen-Friedenreich antigen) on lung, breast and liver cancer-initiating cells. Int J Exp Pathol 92: 97-105, 2011.

21. Livak KJ and Schmittgen TD: Analysis of relative gene expression data using real-time quantitative PCR and the 2(-Delta Delta C (T)) method. Methods 25: 402-408, 2001.

22. Chang HR: Trastuzumab-based neoadjuvant therapy in patients with HER2-positive breast cancer. Cancer 116: 2856-2867, 2010

23. Brändlein S, Pohle T, Ruoff N, Wozniak K, Müller-Hermelink HK and Vollmers HP: Natural IgM antibodies and immunosurveillance mechanisms against epithelial cancer cells in humans. Cancer Res 63: 7995-8005, 2003

24. Axelsson B, Hammarstrom S, Finne J and Perlmann P: The large sialoglycoprotein of human lymphocytes. II. Biochemical features. Eur J Immunol 15: 427-433, 1985.

25. Cermak L, Simova S, Pintzas A, Horejsi V and Andera L: Molecular mechanisms involved in CD43-mediated apoptosis of TF-1 cells. Roles of transcription Daxx expression, and adhesion molecules. J Biol Chem 277: 7955-7961, 2002.

26. Nguyen JT, Evans DP, Galvan M, Pace KE, Leitenberg D, Bui TN and Baum LG: CD45 modulates galectin-1-induced T cell death: regulation by expression of core 2 O-glycans. J Immunol 167: 5697-5707, 2001

27. Hernandez JD, Nguyen JT, He J, et al: Galectin-1 binds different CD43 glycoforms to cluster CD43 and regulate T cell death. J Immunol 177: 5328-5336, 2006.

28. Ma BY, Yoshida K, Baba M, et al: The lectin Jacalin induces human B-lymphocyte apoptosis through glycosylation-dependent interaction with CD45. Immunology 127: 447-488, 2009.

29. Marsters SA, Sheridan JP, Pitti RM, et al: A novel receptor for Apo2L/TRAIL contains a truncated death domain. Curr Biol 7: 1003-1006, 1997.

30. Varambally S, Bar-Dayan Y, Bayry J, et al: Natural human polyreactive IgM induce apoptosis of lymphoid cell lines and human peripheral blood mononuclear cells. Int Immunol 16: 517-524, 2004.

31. Kim HJ, Park HJ, Park WS and Bae Y: CD43 cross-linking increases the Fas-induced apoptosis through induction of Fas aggregation in Jurkat T-cells. Exp Mol Med 38: 357-363, 2006.

32. Kovacs-Solyom F, Blasko A, Fajka-Boja R, et al: Mechanism of tumor cell-induced T-cell apoptosis mediated by galectin-1. Immunol Lett 127: 108-118, 2010.

33. Algeciras-Schimnich A, Shen L, Barnhart BC, Murmann AE, Burkhardt JK and Peter ME: Molecular ordering of the initial signaling events of CD95. Mol Cell Biol 22: 207-220, 2002.

34. Brändlein S, Rauschert N, Rasche L, et al: The human IgM antibody SAM-6 induces tumor-specific apoptosis with oxidized low-density lipoprotein. Mol Cancer Ther 6: 326-333, 2007.

35. Rauschert N, Brändlein S, Holzinger E, Hensel F, MüllerHermelink HK and Vollmers HP: A new tumor-specific variant of GRP78 as target for antibody-based therapy. Lab Invest 88: 375-386, 2008

36. Wagner, KW, Punnoose EA, Januario T, et al: Death-receptor O-glycosylation controls tumor-cell sensitivity to the proapoptotic ligand Apo2L/TRAIL. Nat Med 13: 1070-1077, 2007. 